\title{
Further Laboratory Experiments on Aerosol Scavenging in Mixed Clouds to Assess the Role of Phoretic Forces and Particle Solubility
}

\author{
Franco Prodi' ${ }^{1}$, Gianluca Amirante ${ }^{2}$, Francesco Di Natale ${ }^{2}$, Gianni Santachiara1, Franco Belosi ${ }^{1}$ \\ ${ }^{1}$ Institute of Atmospheric Sciences and Climate, Aerosols, Clouds and Precipitation Group, Bologna, Italy \\ ${ }^{2}$ Department of Chemical Engineering, Federico II University, Naples, Italy \\ Email: f.prodi@isac.cnr.it
}

How to cite this paper: Prodi, F., Amirante, G., Di Natale, F., Santachiara, G. and Belosi, F. (2018) Further Laboratory Experiments on Aerosol Scavenging in Mixed Clouds to Assess the Role of Phoretic Forces and Particle Solubility. Atmospheric and Climate Sciences, 8, 235-247.

https://doi.org/10.4236/acs.2018.82015

Received: November 9, 2017

Accepted: April 27, 2018

Published: April 30, 2018

Copyright $\odot 2018$ by authors and Scientific Research Publishing Inc. This work is licensed under the Creative Commons Attribution International License (CC BY 4.0).

http://creativecommons.org/licenses/by/4.0/

\begin{abstract}
Scavenging experiments have been performed in a cloud chamber inside a cold room with different aerosol particles: Paraffin particles, $\mathrm{NaCl}$ particles, Magnesium oxide particles, Carbon particles, Sahara dust particles. Essentially the experimental tests were carried on following the sequence of operations: the generation of the aerosol particles, their injection in the lower part of the cloud chamber, injection of water droplets in the whole chamber volume, nucleation of ice crystals, collection of ice crystals and their examination as for resulting scavenging efficiency. Evidence is given of the peculiar behaviour of soluble particles, individual and eventually inside mixed particles, leading to very much important scavenging efficiency, probably to be ascribed to aerodynamic capture. The evident peculiar behaviour of deliquescent particles can be oriented towards applications to an efficient abatement of specific effluents, on one side, and to weather modification experiments, both rain enhancement and hail prevention experiments.
\end{abstract}

\section{Keywords}

Aerosol Scavenging, Phoretic Forces, Mixed Clouds

\section{Introduction}

In a review on in-cloud and below cloud scavenging by ice crystals and snowflakes [1] the authors pointed out that past experimental results have been varied and difficult to interpret. The reasons for that have to do with the difficulty in measuring the many parameters involved in such a three component system. The real vapour pressure over ice, over liquid water and in cloud, the tempera- 
ture and water vapour fields, which are determining the competitive role of thermo- and diffusion-phoretic forces, the size distribution and concentration of water droplets injected to grow the crystals and of the crystals themselves during the experiment, the control of ice crystals concentration, the counting of particles once they have been scavenged by ice crystals, the control of electric charges on aerosol particles, on droplets and ice crystals, are among the many parameters involved and conditions to be assessed.

Moreover Brownian diffusion and aerodynamic capture are, or might be, important in their respective domain of size, that of small particles for Brownian and of giant for aerodynamic capture. Electrostatic capture can be effective at all particle sizes and the control of charges, though not so easy, should be mandatory.

On the other side the argument that it is not necessary to perform experiments when we have models are not valid, since models fail in reproducing transient features of many real processes which require a time-dependent description of mechanisms. Instead, models usually treat them as steady phenomena. The group led by a senior author (FP) after a first work [2] which provided evidence of $\mathrm{NaCl}$ particles captured by growing ice crystals, performed experiments both in terrestrial laboratories [3] [4] and in microgravity [5] considered ancillary to solve the scavenging problem. Attention has been given to the role of foretic forces, with thermo- and diffusio-foresis counteracting in the environment of growing or evaporating hydrometeors. The most important outcome from microgravity experiments is considered the one from [5] on aerosols surrounding an evaporating droplet in microgravity conditions. The experiments did show that diffusion-foresis overcomes thermo-foresis (and related Stefan flow) in the aerosol scavenging process. This result is considered a paradigm to evaluate the scavenging efficiency, due to the absence of gravity and related effects, the simple geometry sphere to plane (silica gel plane as a sink of water molecules). Instead more complicated heat and mass transfer rates characterize terrestrial experiments.

Field experiments on in-cloud scavenging [6], among others, or below-cloud scavenging [7], among others on the other side do not solve the problem either, given the difficulties of characterizing the natural conditions as for the role of interstitial aerosol, the electric charges on aerosols, ice crystals and droplets, and eventually the presence of external electric fields.

Prompted by this situation and given the difficulties, listed in [8], of any model in taking into account the transient processes and unsteady situations (oscillations or tumbling of ice crystals etc.) we decided to perform further laboratory experiments to explore a greater variety of parameters and conditions than in [1], and in continuity with [3] taking advantage of improved ability, gained in the meanwhile, in generating and characterizing more aerosol varieties in the laboratory. This in the hope that results of new experiments might enlarge the experimental database available and indicate a direction and strategy for further 
research efforts. In fact an additional expectation from the experiments is that they might suggest lines for a new class of experiments eventually based not on a static chamber but on a continuous flow one, as already suggested by [3], in the line of [9] and [10]. In [3] we can find an additional motivation to resume laboratory scavenging experiments, since electron micrographs were shown of ice crystal replicas with moulded boundaries and cubic $\mathrm{NaCl}$ particles inside suggesting a great role of particle solubility in the process.

In the present work we will present the conditions of the scavenging experiments with the different aerosols, the methods to extract the results and we will discuss them in the light of the role of foretic forces and particle solubility. Finally we will derive suggestions for future experimental configurations.

\section{Experimental Part}

Essentially the experimental tests were carried on following the sequence of operations: the generation of the aerosol particles, their injection in the lower part of the cloud chamber, injection of water droplets in the whole chamber volume, nucleation of ice crystals, collection of ice crystals and their examination as for resulting scavenging efficiency.

\subsection{The Aerosol Generating Systems}

Five aerosols types have been generated in order to explore different parameters involved in the foretic forces: shape, thermal conductivity, hygroscopicity, chemical composition. Both mono-disperse aerosol particles (paraffin) and poly-disperse ( $\mathrm{NaCl}$, Magnesium oxide, Carbon, Sahara dust) have been generated. Different generating systems have been used.

\section{Paraffin particles}

Monodisperse paraffin particles have been generated by the Monodisperse Aerosol GEnerator, MAGE [11] a modified Sinclair-La Mer generator based on controller condensation of the vapours resulting from bubbling in a thermo-stated bath of the substance (carnauba wax or paraffin), then condensing in a heated quartz pipe on embryo particles obtained by drying a diluted $\mathrm{NaCl}$ solution. The required size is obtained by fixing the temperature of the bath and the fraction of the total flux containing the $\mathrm{NaCl}$ embryos. Sizes were $0.25 \mu \mathrm{m}$ and $1.0 \mu \mathrm{m}$ in our tests, with a $\sigma$ of 1.08 and 1.03 respectively.

\section{$\mathrm{NaCl}$ particles}

The generating system is hot wire. A crucible, obtained by a $\mathrm{Ni} / \mathrm{Cr}$ wire spiral in a Pyrex glass jar is filled with salt. The carrier gas flows when the crucible is heated by electric current supplied by a Variac. The aerosol obtained is poly-disperse and the size distribution and shape (transition from cubic to spherical) depend on temperature. The Variac regulator has been kept fixed at $25 \mathrm{~V} / \mathrm{A}$ in order to have the same size distribution in all tests. Flow rate has been regulated to optimize the particle concentration, not too low to make the test effective and not too high to have significant coagulation. 
Magnesium oxide particles

Magnesium oxide particles have been generated by combustion of a thin strip of magnesium metal according to the reaction

$$
\mathrm{Mg}_{(\mathrm{s})}+\mathrm{O}_{2(\mathrm{~g})} \rightarrow \mathrm{MgO}_{(\mathrm{s})}
$$

The combustion takes place in a glass jar with an entrance and exit for the flow of carrier gas. $\left(\mathrm{N}_{2}\right)$. The same pump as for $\mathrm{NaCl}$ aerosol was used.

$\mathrm{Mg}$ aerosol particles, non oxide, can be obtained by discharge between electrodes as the following case of carbon, but we did not generate this type of aerosol.

\section{Carbon particles}

The carbon aerosol has been generated by discharge between electrodes. Two graphite electrodes are at close distance $(2 \mathrm{~mm})$ in a glass jar with two openings for entrance and exit of the carrier gas. With this system particles of size of the order of hundreds of nano-meters are obtained. Due to the low concentrations that can be obtained in this way we reduced the flow rate and extended the generation time until the required concentration is obtained.

Sahara dust particles

A sample of Sahara dust particles collected after being deposited on a clean substrate during a muddy rain event has been aerosolized by means of a cyclone in a flow of dry air. The sample is characterized in size distribution and composition in [12].

\subsection{The Cloud Chamber}

The experiment has been performed with a static cloud chamber similar as the one described in [2], except the vertical superposition of two modules separated by a sliding sheet. Each module was a perspex cylinder $(22 \mathrm{~cm}$ in diameter, 96 $\mathrm{cm}$ in height and 36.5 liters capacity) placed in a cold room, the temperature of which could be regulated down to $-28^{\circ} \mathrm{C}$. On top an opening is left for liquid nitrogen cooled rod entering to nucleate ice crystals. More openings in the body allow for the injection of aerosols and water droplets, or sampling internal air for aerosol concentration measurements. In the bottom plane an opening with a sled allows microscope glass slides to be inserted and exposed for falling ice crystal collection and replication without changing the conditions inside the chamber. In Figure 1 the column is shown.

\subsection{Sequence of Operations during Each Test}

The bag filled with aerosol generated in the warm laboratory is moved in the cold laboratory and after a proper time for temperature stabilization, is injected in the lower volume of the chamber. Water droplets are injected in both volumes, with the sliding sheet closed. Droplets in the upper volume are nucleated to ice by the liquid nitrogen cooled rod, from the top. After a time long enough for the tiny crystals to fall, the sliding sheet is opened and the crystals are further fed by the water droplets in the lower volume and settle on the bottom, on the 
(a)

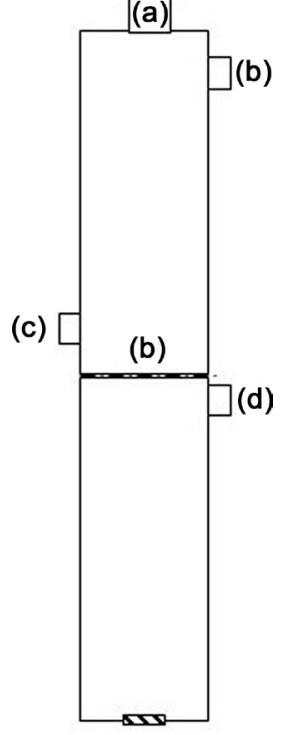

(e)

Figure 1. A scheme of the column used: (a) the nucleation zone; (b) sliding sheet; (c) higher water input zone; (d) lower water and aerosol input zone and (e) microscope glass slide for replicating falling crystals. Each module of the chamber was a perspex cylinder (22 cm in diameter, $96 \mathrm{~cm}$ in height and 36.5 liters capacity).

microscope slide covered with formvar solution. The formvar solution is a $2 \%$ solution of polyvinyl formal in chloroform. The solution is spread on the glass slide before it is inserted in the slid. The glass slides with ice crystals deposited are kept in a glass jar to prevent water condensation when in the warm laboratory, left to dry and then observed at optical microscope and SEM for collected particles. All operations are voice recorded for a correct timing in the post processing.

\subsection{Assessment of Scavenging Efficiency}

The glass slides are examined at the optical microscope to locate the best areas and the corresponding glass portions are glued on a stub for SEM observations. Then the scavenging efficiency is directly evaluated from the pictures. An indirect estimate was attempted by measuring particle concentration in the chamber by a dust track, with non conclusive results due to ice crystals and droplets interference.

\section{Results}

\section{Paraffin particles}

Tests have been performed with $0.2 \mu \mathrm{m}$ particle size. Not effective scavenging was noticed.

With $1.0 \mu \mathrm{m}$ size few more particles were observed, possibly due to aerodynamic capture initiating to be effective.

\section{$\mathrm{NaCl}$ particles}

Rounded boundaries crystals with a cascade of particles exiting is a striking result, shown in Figures 2(a)-(d). The particles are inside the replica, with 


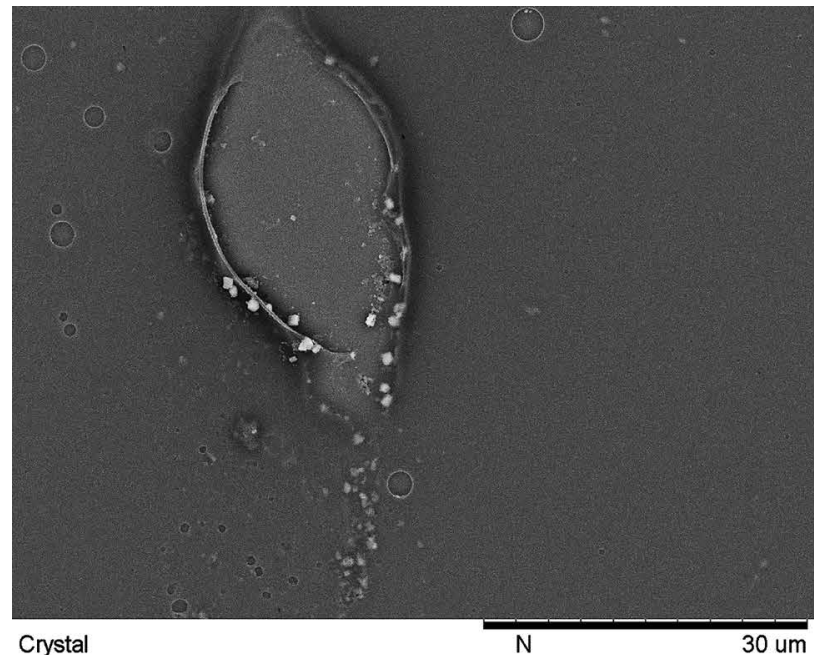

(a)

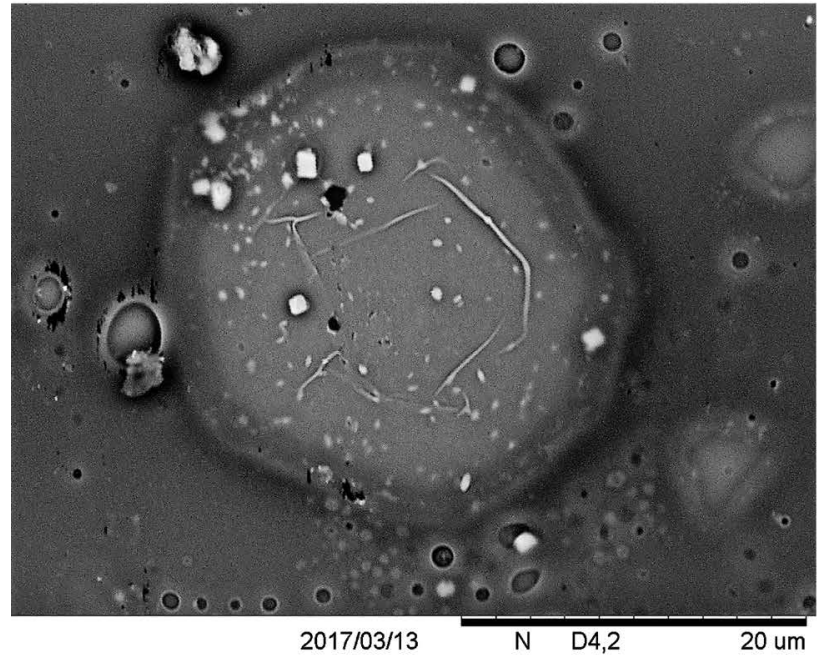

(c)

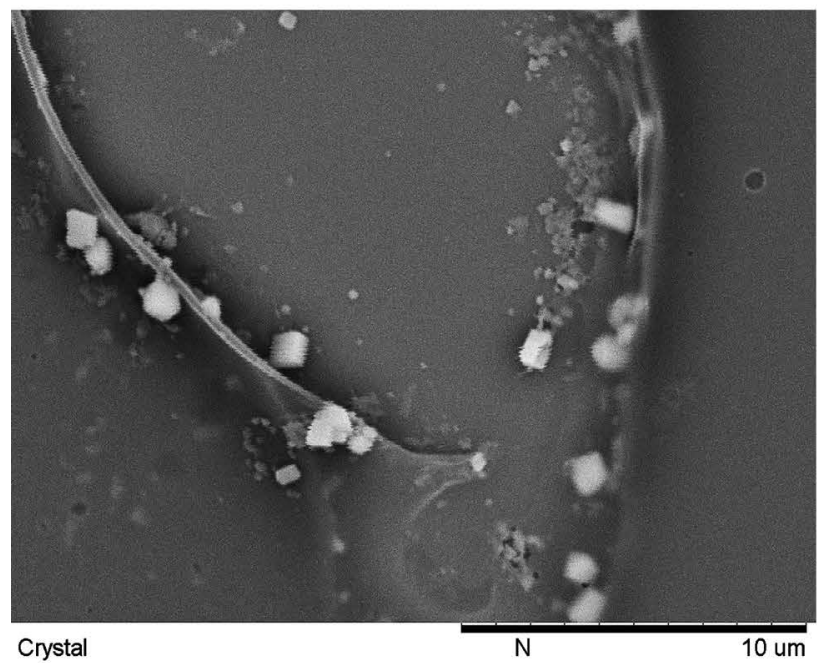

(e)

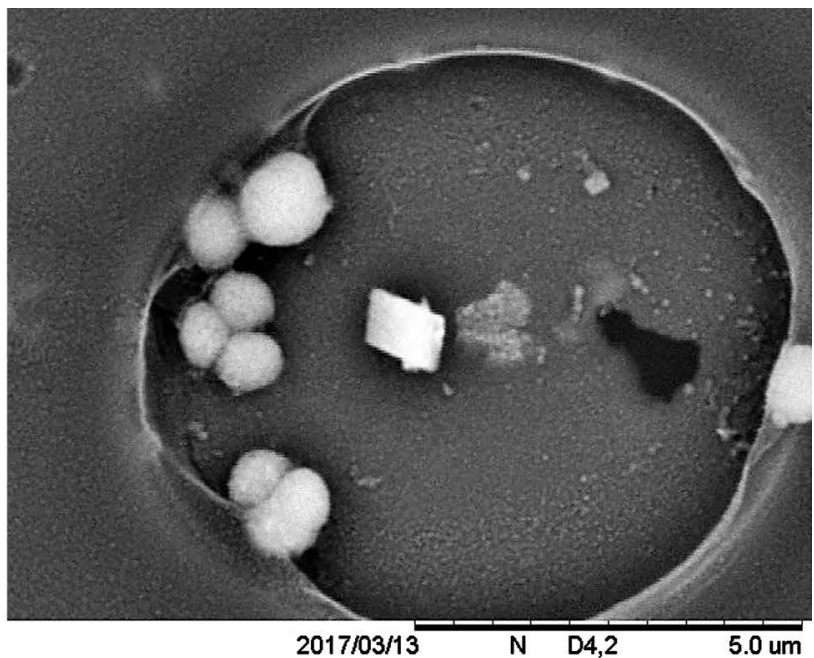

(b)

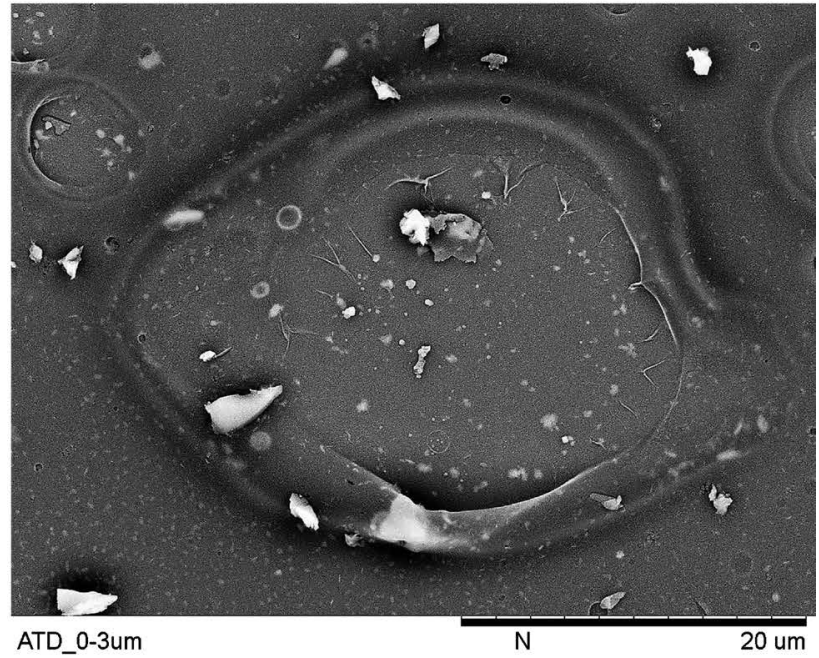

(d)

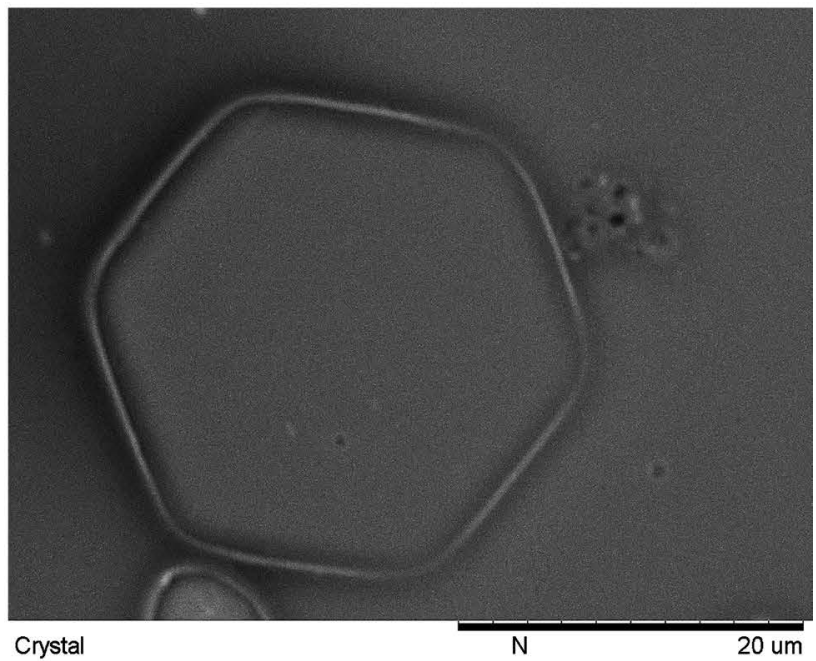

(f)

Figure 2. (a)-(d): Cascade of particles of $\mathrm{NaCl}$ in some replicas; (e) Particular of the particles shown in Figure 2(a), they are greater respect their original size; (f) The crystals that haven't captured particles in the same test have the original hexagonal shapes. 
rounded boundaries, and flowing out of it. The difference from the original particles sizes is evident in Figure 2(e). Though the efficiency of scavenging is apparent, other crystals maintain their distinctive hexagonal shapes without captured particles (see Figure 2(f) of the same test). The first feature, moulded crystal replicas with remarkable numbers of scavenged large $\mathrm{NaCl}$ particles, is dominant.

We anticipate that the role of particle solubility and deliquescence is absolutely crucial. Figure 2(a) with its magnified detail of Figure 2(e) show that the moulding process is taking place while the crystal is falling, otherwise the replica would show sharp crystal boundaries. The formvar film is so thin that in this case a flow of particles exit outside, after breaking the film. The particles exhibit a typical $\mathrm{NaCl}$ cubic-like shape, but with larger sizes than the originally generated particles. There is a complete rearrangement in favour of larger sizes. It looks as a solution drop-crystal mixture with high solute concentration, as a result of final process of evaporation, gives a distribution of its own. Figure 2(b) shows spherical $\mathrm{NaCl}$ particles similar to those we obtain when the hot wire of the crucible is at high temperatures. The reason for having spherical instead cubic shape as a result of the same process should be investigated. Figure $2(\mathrm{c})$ has no peculiarity; the large cubic $\mathrm{NaCl}$ particles do not break the formvar film and stay inside. Particular care should be give in considering Figure 2(d) as regard the spiky particle, which are artefacts as glass slide fragments during the operation of adjusting the glass piece on the stub. Tiny $\mathrm{NaCl}$ particles inside the moulded crystal replica can again be observed.

Magnesium oxide particles

Tests with MgO particles show evidence of scavenging effects but only at a given stage of the growth. Boundaries are distinctive, not rounded, with the most of the particles on them. Effect of the deliquescence is not appreciable at all. Actually $\mathrm{MgO}$ particles are not supposed to be deliquescent. Figures representative of these results are Figure 3(a) for all the crystal and Figure 3(b) for the detail of a corner. A large crystal, free of particles, is shown in Figure 4(a) and detail of particles detected in a branch is shown in Figure 4(b).

Carbon particles

No particles are detected in ice crystal replicas.

Sahara dust particles

Larger crystals captured both small and larger particles, as shown in Figure 5(a), but in relativity small numbers. Smaller crystals capture submicron particles, smaller than $0.8 \mu \mathrm{m}$, preferably localised nearby the borders as shown in Figure $5(\mathrm{~b})$ where the roof of replica collapse on the particles which are relieved by the relieves of the roof itself.

In this test we have done sizing and the captured particles counting in different crystals. The counting has been performed on larger crystals, that are deposited in the first part of the experiment, and on smaller crystals deposited at the end.

The results are reported in Table 1. 


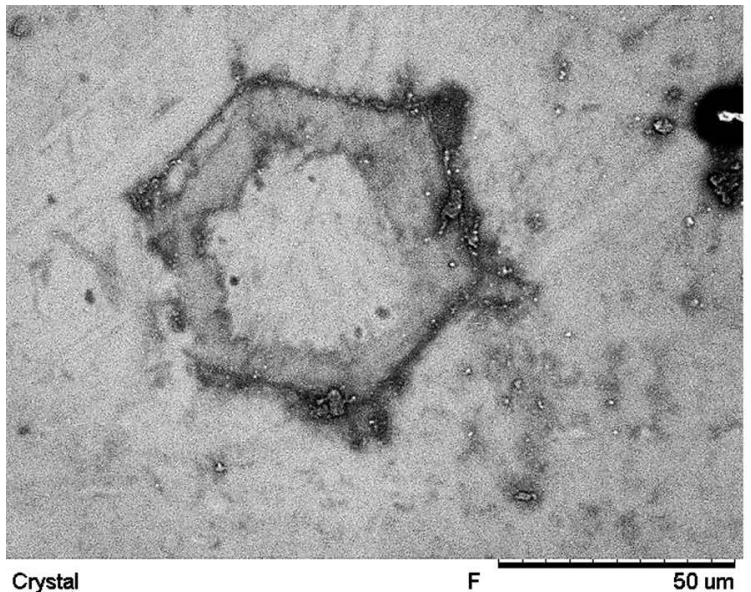

(a)

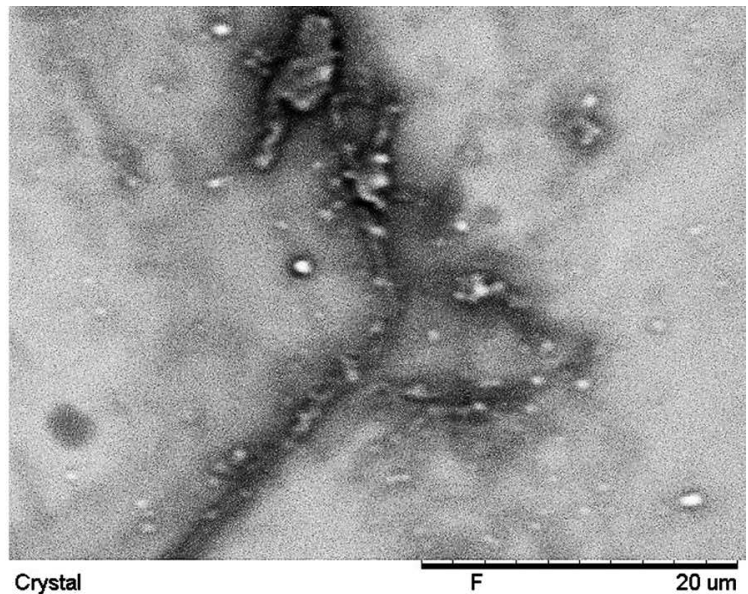

(b)

Figure 3. A crystal whit $\mathrm{MgO}$ particles around the boundaries, it is shown how the particles that are captured are localized especially in the corners of the crystal.

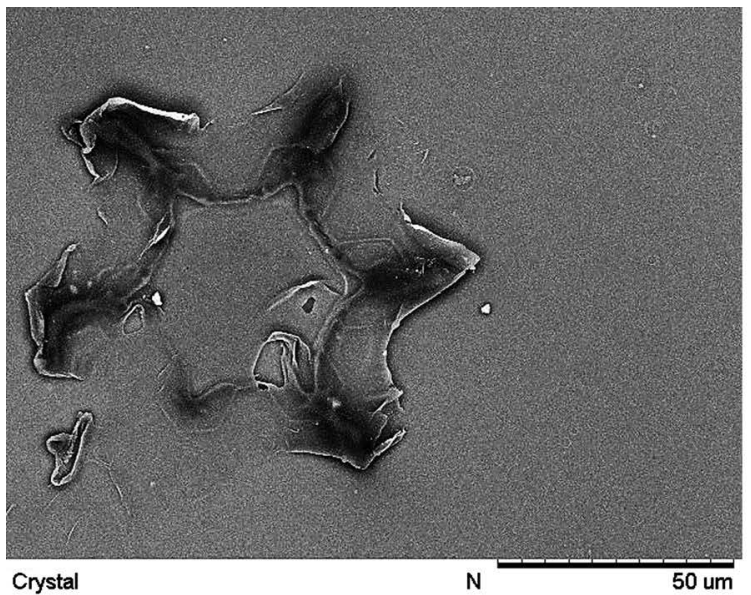

(a)

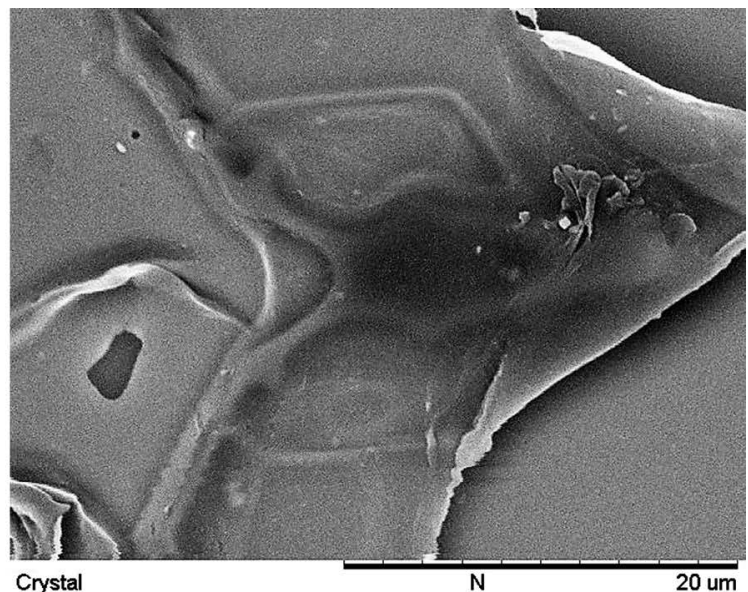

(b)

Figure 4. A large crystal replica, it is free of particles. Is possible to see how the particles are captured only in the area of the boundaries of the crystal.

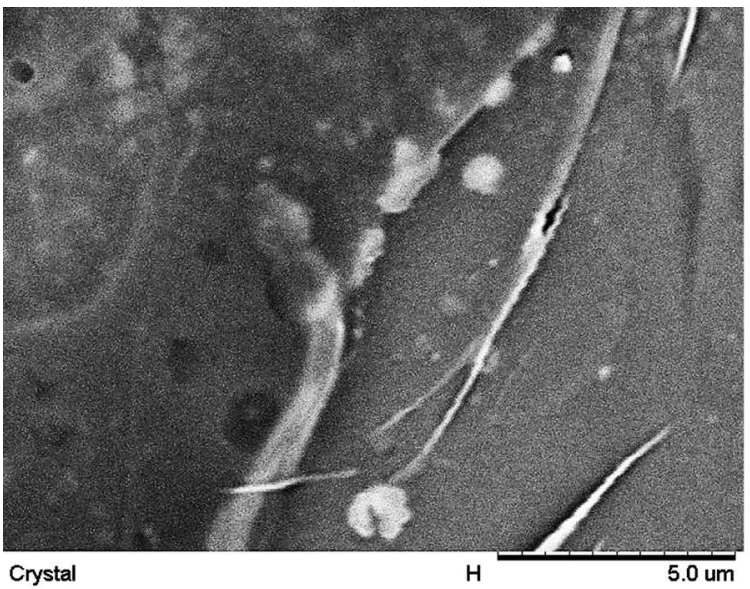

(a)

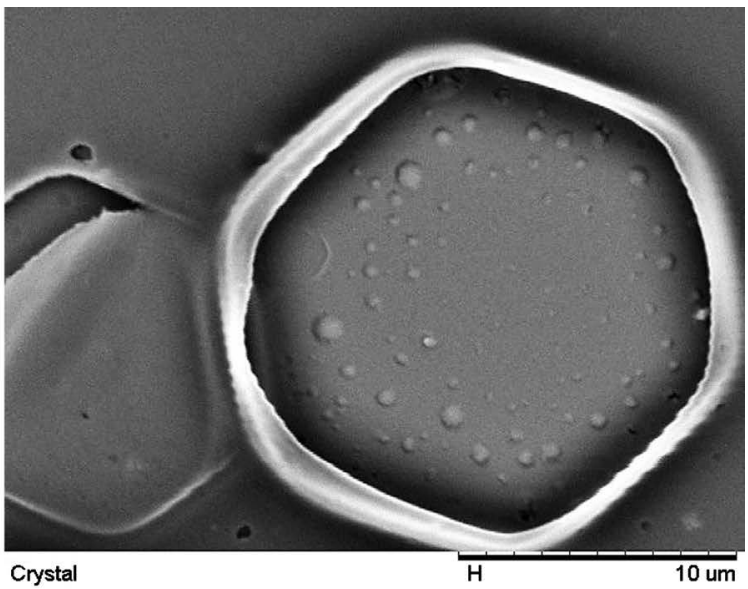

(b)

Figure 5. (a) Particles of Sahara dust in a large crystal $(150 \mu \mathrm{m})$; (b) Particles captured by a small crystal, they are localized under a roof of Formvar. 
Table 1. Number of aerosol particles captured for different ice crystal diameter. dc is diameter of crystals.

\begin{tabular}{ccccc}
\hline \multirow{2}{*}{$\mathrm{dc}[\mu \mathrm{m}]$} & \multicolumn{4}{c}{ Captured particle diameter } \\
\cline { 2 - 5 } & $\mathbf{0 - 0 . 4}$ & $\mathbf{0 . 4 - 0 . 8}$ & $\mathbf{0 . 8}-\mathbf{>}$ \\
\hline $\mathbf{2 0}$ & 3 & 7 & 0 & 0 \\
30 & 2 & 8 & 3 & 0 \\
45 & 3 & 10 & 4 & 0 \\
90 & 8 & 14 & 8 & 10 \\
130 & 6 & 12 & 7 & 12 \\
150 & 4 & 9 & 6 & 12 \\
160 & 4 & 5 & 4 & 7 \\
180 & 3 & 5 & 8 & 13 \\
\hline
\end{tabular}

\section{Discussion}

When an experiment is planned and carried on the results cannot be foreseen, we are searching for them, and in a certain way we are ready to be surprised by them. In the results presented in the previous section the most relevant surprise is the striking role of the deliquescent particles in the scavenging process, in presence of growing crystals. In fact with $\mathrm{NaCl}$ poly-disperse particles as aerosols we find on the glass slide at the bottom of the chamber replicas of moulded crystals which almost lost their original neat shape, with cubic $\mathrm{NaCl}$ particles clearly not related in size to the original distribution, when they were injected from the bag into the lower volume of the chamber. It is natural to discuss first this result, unexpected with such evidence.

Occasionally the discussion is carried on by comparison with [3], though no dynamic chamber investigation and testing has been performed here. Carnauba wax in [3] and paraffin particles, essentially sharing similar physical characteristics behave in the same way, with low scavenging efficiency. So there is no reason to discuss further this point, on paraffin particles.

Then we will discuss the results concerning other, non soluble, aerosols. Finally starting from the shortcomings or weakness of the present experiments we will attempt to give indications and suggestions for further experiments.

1) Deliquescent $\mathrm{NaCl}$ particles in scavenging

In [3] $\mathrm{NaCl}$ particles remained small in small crystals maybe for $\mathrm{U}_{\text {rel }}$ less than $45 \%$ almost no collection in evaporating crystals.

For temperatures above the eutectic point the solution destroys the sharp boundaries and indicate a rounded almost melted crystals.

In our experiment we know approximately well what we inject: poly-disperse water droplets and poly-disperse $\mathrm{NaCl}$ particles. When opening the slid we let ice crystals to fall and grow, then, we know what we find on the glass slide. We can only speculate on what is happening in the meanwhile. Let us be guided, among the many papers in the literature on deliquescent particles by the clear 
and summarizing chapter by [13] devoted to the deliquescence and growth of mixed salt particles relating aerosol phase transformation to the solubility diagrams for multi-component electrolyte solutions. From the growth and evaporation cycles at $25^{\circ} \mathrm{C} \mathrm{NaCl}$ particles deliquesce at $75.5 \% \mathrm{RH}$ and crystallizes again at $48 \% \mathrm{RH}$. Moreover it is to be noticed that a single particle can stay as a solid or as a droplet, cannot stay in partial dissolution stage.

Soon after the injection of the two separate components we will have $\mathrm{NaCl}$ particles changing into droplets with water droplets contributing to their growth by partial evaporation via the environmental water vapour. This is the environment that ice crystals encounter during fall with pure water droplets providing vapour by diffusion towards the ice crystals, and solution droplets doing the same but along trajectories pertaining to their new sizes. The problem is rather complicated since it would imply the reconstruction of the resulting water vapour field. The sequence would see a population of $\mathrm{NaCl}$ solution droplets being more easily captured on the crystal surface by aerodynamic capture. Once the $\mathrm{NaCl}$ droplet reaches the ice crystal surface it spreads on the surface generating a liquid film, being above the eutectic point. Captured in numbers, they mould the boundaries and partially melt the ice crystal. The resulting replica shape is the one shown in Figures 2(a)-(e), but the next efflorescence stage is mediated by the large liquid content of the melting crystal, thus giving origin to particles not at all related to the size distribution of the originally injected particles. This process needs to be modelled and we would find the scavenging via deliquescence a very effective mechanisms and this could also explain the fact that we find fallen later crystals with neat boundaries and free of particles as in Figure 2(f) by not finding any more solution droplets in their way.

So we can say that $\mathrm{NaCl}$ main result of [3] confirmed, but more evidence has been given to the importance of particle deliquescence in the scavenging process.

It could be speculated that solution droplet evaporation in vicinity of the crystal cool the droplet and generate efflorescence "in flight" Vapour pressure on the three components should be modelled in the process.

This mechanism of deliquescent scavenging seems so effective that applications can be sought for, both in abatement of polluted effluents and in weather modification experiments.

We add a note on mixed particles. We have used almost pure salts but we know that the growth of atmospheric aerosol particles in continental atmosphere deviates substantially from what is predicted from growth of pure salts. In mixed particles soluble part exhibit meta-stable behaviour between 45 and 75\% RH. Solution droplets tend to become highly supersaturated before efflorescence. Hydration properties of hygroscopic aerosol particles cannot be predicted from the bulk solution properties [13]. Upon evaporation the solution droplets become highly supersaturated until crystallization occurs at about $58 \% \mathrm{RH}$ to anhydrous particles. [14] has also considered phase transformation and droplet growth in mixed-salt aerosols, relating aerosol phase transformation and growth 
to the solubility diagrams for multiple component electrolyte solutions. The question arise about which equilibrium between an aqueous salt solution and water vapour in humid air at a given $\mathrm{T}$ and $\mathrm{RH}$.

2) $\mathrm{MgO}$ particles

The results on $\mathrm{MgO}$, distinctive with respect to deliquescent $\mathrm{NaCl}$, can be explained on the formation of a thin liquid film on the particle leading to Magnesium hydroxide, via an hydration mechanism. Due to the low solubility the hydroxide acts as a skin hindering the particle from further absorbing water. This according to [15] who consider the following steps: water vapour is first absorbed chemically on $\mathrm{MgO}$ surface to form a liquid layer, which reacts with $\mathrm{MgO}$ to form $\mathrm{Mg}(\mathrm{OH})_{2}$ layer This would explain the different behaviour with respect to $\mathrm{NaCl}$ particles.

3) Other non soluble particles: $C$ and Sahara dust particles

Results for non soluble aerosols confirm results of [3] of scanty scavenging efficiency. Though not using the same monodisperse particles (paraffin in place of carnauba wax) in the MAGE, thermal conductivity is not much different. On the other side comparison with results of [10] are difficult for the different experimental conditions in temperature, liquid water content and ice crystal diameters. Moreover Sahara dust particles being of natural origin (collected as they were in muddy rain in Sestola, northern Italian Appennines) might contain also mixed particles with soluble component.

4) Possible experimental improvements

We noticed the difficulties in having a complete control of experimental parameters in a three component system, with transient features. When the slid is open separating the two chamber volumes the ice crystals nucleated in the upper part fall after having reached different sizes and encounter an environment they contribute to change. The front crystals interact with it either by depriving the surrounding water vapour causing water droplets to evaporate and leaving decreased aerosol concentrations, having eventually scavenged part of the particles. A strategy for future scavenging experiments should be based on a continuous flow chamber with accurate design in regulating updraft velocity, the flows of super-cooled droplets and aerosol, and a carefully controlled ice nucleating process.

\section{Conclusions}

A laboratory study of aerosol scavenging by ice crystals in super-cooled cloud has been carried on, to widen the experimental database. However we share the same comment of [10] that is not simple to compare the results of different experiments, to the point that they preferred to compare them with model results, not to experiments.

However we

- tried to make use of the experimental results obtained in the meanwhile in microgravity conditions about the competitive role of foretic forces around 
an evaporating droplet;

- gave evidence of the peculiar behaviour of soluble particles, individual and eventually inside mixed particles, lead to very much important scavenging efficiency, probably to be ascribed to aerodynamic capture;

- this evident peculiar behaviour of deliquescent particles can be oriented towards applications to and efficient abatement of specific effluents, on one side, and to weather modification experiments, both in rain enhancement and hail prevention experiments-indications for future experiments are obtained in requirements of continuous flow chambers or the sophisticated cloud chamber facilities now in operation in USA, UK, Germany and Japan;

- it is evident that laboratory experiments as those presented here, though carefully performed, cannot answer all questions in the intriguing field of the removal mechanisms of atmospheric particles from the atmosphere. Our series of paper on foretic forces in microgravity conditions helped clarify the specific roles of thermo and diffusion-foresis, but Brownian capture, electrostatic capture and aerodynamic one should be combined to produce a coherent picture.

\section{Acknowledgements}

The technical assistance of G. Trivellone is gratefully acknowledged.

\section{References}

[1] Santachiara, G., Prodi, F. and Belosi, F. (2013) Atmospheric Aerosol Scavenging Processes and the Role of Thermo- and Diffusion-Phoretic Forces. Atmospheric Research, 128, 46-56. https://doi.org/10.1016/j.atmosres.2013.03.004

[2] Prodi, F. (1976) Scavenging of Aerosol Particles by Growing Ice Crystals. Prepr. Int. Conference on Cloud Physics, Boulder, 26-30 July 1976, 70-75.

[3] Prodi, F. and Oraltay, R.G. (1991) Scavenging of Aerosol Particles by Growing and Evaporating Ice Crystals: Assessing the Role of Competitive Processes. Proc. 5th Int.Conf. on Precipitation Scavenging and Atmosphere-Surface Exchange Processes, Richland, 15-19 July 1991, 75-86.

[4] Santachiara, G., Prodi, F. and Buzzoni, N. (2000) Scavenging of Aerosols by Growing Ice Crystals Obtained with Control of Electrical Conditions. Proc.13th Conf. Clouds and Precipitation, Reno, 14-18 August 2000, 59-62.

[5] Prodi, F., Santachiara, G., Belosi, F., Vedernikov, A. and Balapanov, D. (2014) Foretic Forces on Aerosol Particles Surrounding an Evaporating Droplet in Microgravity Conditions. Atmospheric Research, 142, 40-44. https://doi.org/10.1016/j.atmosres.2013.09.001

[6] Schwikowski, M., Baltensperger, U., Gaggeler, H.W. and Poulida, O. (1998) Scavenging of Atmospheric Constituents in Mixed Phase Clouds at the High-Alpine Site Jungfraujoch. Part III. Quantification of the Removal of Chemical Species by Precipitating Snow. Atmospheric Environment, 23, 4001-1010. https://doi.org/10.1016/S1352-2310(98)00050-8

[7] Murakami, M., Hiramatsu, C. and Magono, C. (1981) Observations of Aerosol Scavenging by Falling Snow Crystals at Two Sites of Different Heights. Journal of the Meteorological Society of Japan, 59, 763-771. 
https://doi.org/10.2151/jmsj1965.59.5_763

[8] Prodi, F. and Tampieri, F. (1982) The Removal of Particulate Matter form the Atmosphere: The Physical Mechanisms. PAGEOPH, 120, 286-325.

https://doi.org/10.1007/BF00877038

[9] Song, N. and Lamb D. (1994) Experimental Investigation of Ice in Super-Cooled Clouds. Part I: System Description and Growth of Ice by Vapour Deposition. Journal of the Atmospheric Sciences, 51, 91-103. https://doi.org/10.1175/1520-0469(1994)051<0091:EIOIIS >2.0.CO;2

[10] Song, N. and Lamb D. (1994) Experimental Investigation of Ice in Super-Cooled Clouds Part II: Scavenging of an Insoluble Aerosol. Journal of the Atmospheric Sciences, 51, 104-116. https://doi.org/10.1175/1520-0469(1994)051<0104:EIOIIS>2.0.CO;2

[11] Prodi, V. (1972) A Condensation Aerosol Generator for Solid Monodisperse Particles. In: Mercer, T.T., Morrow, P.P. and Stober, W., Eds., Assessment of Airborne Particles, Thomas Publisher, 169-181.

[12] Prodi, F. and Fea, G. (1979) A Case of Transport and Deposition of Saharan Dust over Italian Peninsula and Southern Europe. Journal of Geophysical Research, 84, C11. https://doi.org/10.1029/JC084iC11p06951

[13] Tang, I.N. (2000) Phase Transformation and Growth of Hygroscopic Aerosols. In: Spurny, K.R., Ed., Aerosol Chemical Processes in the Environment, CRC Press LLC., Boca Raton, 6951-6960. https://doi.org/10.1201/9781420036251.ch4

[14] Tang, I.N. (1976) Phase Transformation and Growth of Aerosol Particles Composed of Mixed Salts. Journal of Aerosol Science, 7, 361. https://doi.org/10.1016/0021-8502(76)90022-7

[15] Smithson, G.L. and Bakhashi, N.N. (1969) The Kinetics and Mechanism of the Hydration of Magnesium Oxide in a Batch Reactor. Canadian Journal of Chemical Engineering, 47, 508-513. https://doi.org/10.1002/cjce.5450470602 